\title{
Relational String Verification Using Multi-track Automata *
}

\author{
Fang Yu, Tevfik Bultan, and Oscar H. Ibarra \\ Department of Computer Science, University of California, Santa Barbara, CA, USA \\ \{yuf, bultan, ibarra\}@es.ucsb.edu
}

\begin{abstract}
Verification of string manipulation operations is a crucial problem in computer security. In this paper, we present a new relational string verification technique based on multi-track automata. Our approach is capable of verifying properties that depend on relations among string variables. This enables us to prove that vulnerabilities that result from improper string manipulation do not exist in a given program. Our main contributions in this paper can be summarized as follows: (1) We formally characterize the string verification problem as the reachability analysis of string systems and show decidability/undecidability results for several string analysis problems. (2) We develop a sound symbolic analysis technique for string verification that over-approximates the reachable states of a given string system using multi-track automata and summarization. (3) We evaluate the presented techniques with respect to several string analysis benchmarks extracted from real web applications.
\end{abstract}

\section{Introduction}

The most important Web application vulnerabilities are due to inadequate manipulation of string variables [10]. In this paper we investigate the string verification problem: Given a program that manipulates strings, we want to verify assertions about string variables. For example, we may want to check that at a certain program point a string variable cannot contain a specific set of characters. This type of checks can be used to prevent SQL injection attacks where a malicious user includes special characters in the input string to inject unintended commands to the queries that the Web application constructs (using the input provided by the user) and sends to a backend database. As another example, we may want to check that at a certain program point a string variable should be prefix or suffix of another string variable. This type of checks can be used to prevent Malicious File Execution (MFE) attacks where Web application developers concatenate potentially hostile user input with file functions that lead to inclusion or execution of untrusted files by the Web server.

We formalize the string verification problem as reachability analysis of string systems (Section 2). After demonstrating that the string analysis problem is undecidable in general, we present and implement a forward symbolic reachability analysis technique that computes an over-approximation of the reachable states of a string system using widening and summarization (Section 4). We use multi-track deterministic finite

\footnotetext{
* This work is supported by NSF grants CCF-0916112, CCF-0716095, and CCF-0524136.
} 
automata (DFAs) as a symbolic representation to encode the set of possible values that string variables can take at a given program point. Unlike prior string analysis techniques, our analysis is relational, i.e., it is able to keep track of the relationships among the string variables, improving the precision of the string analysis and enabling verification of invariants such as $X_{1}=X_{2}$ where $X_{1}$ and $X_{2}$ are string variables. We develop the precise construction of multi-track DFAs for linear word equations, such as $c_{1} X_{1} c_{2}=c_{1}^{\prime} X_{2} c_{2}^{\prime}$ and show that non-linear word equations (such as $X_{1}=X_{2} X_{3}$ ) cannot be characterized precisely as a multi-track DFA (Section 3). We propose a regular approximation for non-linear equations and show how these constructions can be used to compute the post-condition of branch conditions and assignment statements that involve concatenation. We use summarization for inter-procedural analysis by generating a multi-track automaton (transducer) characterizing the relationship between the input parameters and the return values of each procedure (Section 4). To be able to use procedure summaries during our reachability analysis we align multi-track automata so that normalized automata are closed under intersection. We implemented these algorithms using the MONA automata package [5] and analyzed several PHP programs demonstrating the effectiveness of our string analysis techniques (Section 5).

Related Work. The use of automata as a symbolic representation for verification has been investigated in other contexts [4]. In this paper, we focus on verification of string manipulation operations, which is essential to detect and prevent crucial web vulnerabilities. Due to its importance in security, string analysis has been widely studied. One influential approach has been grammar-based string analysis that statically computes an over-approximation of the values of string expressions in Java programs [6] which has also been used to check for various types of errors in Web applications [8,9, 12]. In $[9,12]$, multi-track DFAs, known as transducers, are used to model replacement operations. There are also several recent string analysis tools that use symbolic string analysis based on DFA encodings $[7,11,14,15]$. Some of them are based on symbolic execution and use a DFA representation to model and verify the string manipulation operations in Java programs $[7,11]$. In our earlier work, we have used a DFA based symbolic reachability analysis to verify the correctness of string sanitization operations in PHP programs [13-15]. Unlike the approach we proposed in this paper, all of the results mentioned above use single track DFA and encode the reachable configurations of each string variable separately. Our multi-track automata encoding not only improves the precision of the string analysis but also enables verification of properties that cannot be verified with the previous approaches.

We have also investigated the boundary of decidability for the string verification problem. Bjørner et al. [2] show the undecidability result with replacement operation. In this paper we consider only concatenation and show that string verification problem is undecidable even for deterministic string systems with only three unary string variables and non-deterministic string systems with only two string variables if the comparison of two variables are allowed.

\section{String Systems}

We define the syntax of string systems in Figure 1. We only consider string variables and hence variable declarations need not specify a type. All statements are labeled. We 


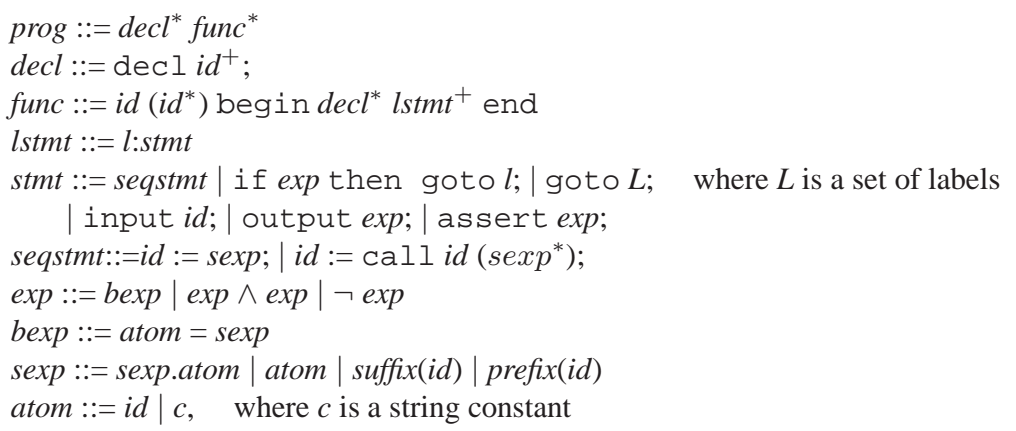

Fig. 1. The syntax of string systems

only consider one string operation (concatenation) in our formal model; however, our symbolic string analysis techniques can be extended to handle complex string operations (such as replacement [14]). Function calls use call-by-value parameter passing. We allow goto statements to be non-deterministic (if a goto statement has multiple target labels, then one of them is chosen non-deterministically). If a string system contains a non-deterministic goto statement it is called a non-deterministic string system, otherwise, it is called a deterministic string system.

There are several attributes we can use to classify string systems such as deterministic $(D)$ or non-deterministic $(N)$ string systems, the number of variables in the string systems, and the alphabet used by the string variables, e.g., unary $(U)$, binary $(B)$, or arbitrary $(K)$ alphabet. Finally, we can restrict the set of string expressions that can be used in the assignment and conditional branch instructions. As an instance, $N B\left(X_{1}, X_{2}\right)_{X_{1}=X_{2}}^{X_{i}:=X_{i} c}$ denotes a non-deterministic string system with a binary alphabet and two string variables $\left(X_{1}\right.$ and $\left.X_{2}\right)$ where variables can only concatenate constant strings from the right and compared to each other. We use $a$ to denote a single symbol, and $c, d$ to denote constant strings. $c=\operatorname{prefix}\left(X_{i}\right)$ evaluates to true if $c$ is a prefix of $X_{i}$, and $c=\operatorname{suffix}\left(X_{i}\right)$ evaluates to true if $c$ is a suffix of $X_{i}$. We define the reachability problem for string systems is the problem of deciding, given a string system and a configuration (i.e., the instruction label and the values of the variables), whether at some point during a computation, the configuration will be reached. We have the following results:

Theorem 1. The reachability problem for:

$$
\begin{aligned}
& \text { 1. } N B\left(X_{1}, X_{2}\right)_{X_{1}=X_{2}}^{X_{i}:=X_{i} c} \text { is undecidable, } \\
& \text { 2. } D U\left(X_{1}, X_{2}, X_{3}\right)_{X_{1}=X_{3}, X_{2}=X_{3}} \text { is undecidable, } \\
& \text { 3. } D U\left(X_{1}, X_{2}, X_{3}, X_{4}\right)_{X_{1}=X_{3}, X_{2}=X_{4}}^{X_{i}, X_{i}} \text { is undecidable, }
\end{aligned}
$$

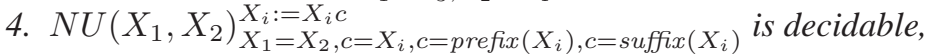

$$
\begin{aligned}
& \text { 5. } N K\left(X_{1}, X_{2}, \ldots, X_{k}\right)_{c=X_{i}, c=p r e f i x}^{\left.X_{i}:=d X_{i}\right), c=\operatorname{suffix}\left(X_{i}\right)} \text { is decidable, and }
\end{aligned}
$$

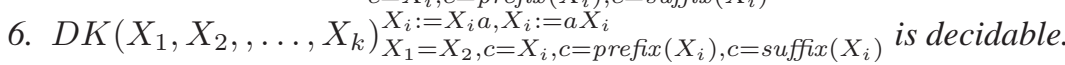

Theorem 1 demonstrates the complexity boundaries for verification of string systems. Theorem 1.1, 1.2 and 1.3 show that the string verification problem can be unde- 
cidable even when we restrict a non-deterministic string system to two binary variables, or a deterministic string system to three unary variables or four unary variables with specific comparisons. Theorem 1.4 shows that the three variables in Theorem 1.2 are necessary in the sense that when there are only two variables, reachability is decidable, even when the string system is nondeterministic. Theorem 1.5 and 1.6, on the other hand, demonstrate that there are non-trivial string verification problems that are decidable. Since the general string verification problem is undecidable, it is necessary to develop conservative approximation techniques for verification of string systems. In this paper we propose a sound string verification approach based on symbolic reachability analysis with conservative approximations where multi-track automata are used as a symbolic representation. Some examples of string systems that can be verified using our analysis are given in [16].

\section{Regular Approximation of Word Equations}

Our string analysis is based on the following observations: (1) The transitions and the configurations of a string system can be symbolically represented using word equations with existential quantification, (2) word equations can be represented/approximated using multi-track DFAs, which are closed under intersection, union, complement, and projection, and (3) the operations required during reachability analysis (such as equivalence checking) can be computed on DFAs.

Multi-track DFAs A multi-track DFA is a DFA but over the alphabet that consists of many tracks. An $n$-track alphabet is defined as $(\Sigma \cup\{\lambda\})^{n}$, where $\lambda \notin \Sigma$ is a special symbol for padding. We use $w[i](1 \leq i \leq n)$ to denote the $i^{t h}$ track of $w \in(\Sigma \cup\{\lambda\})^{n}$. An aligned multi-track DFA is a multi-track DFA where all tracks are left justified (i.e., $\lambda$ 's are right justified). That is, if $w$ is accepted by an aligned $n$-track DFA $M$, then for $1 \leq i \leq n, w[i] \in \Sigma^{*} \lambda^{*}$. We also use $\hat{w}[i] \in \Sigma^{*}$ to denote the longest $\lambda$-free prefix of $w[i]$. It is clear that aligned multi-track DFA languages are closed under intersection, union, and homomorphism. Let $M_{u}$ be the aligned $n$-track DFA that accepts the (aligned) universe, i.e., $\left\{w \mid \forall i . w[i] \in \Sigma^{*} \lambda^{*}\right\}$. The complement of the language accepted by an aligned $n$-track DFA $M$ is defined by complement modulo alignment, i.e., the intersection of the complement of $L(M)$ with $L\left(M_{u}\right)$. For the following descriptions, a multi-track DFA is an aligned multi-track DFA unless we explicitly state otherwise.

Word Equations A word equation is an equality relation of two words that concatenate variables from a finite set $\mathbf{X}$ and words from a finite set of constants $\mathcal{C}$. The general form of word equations is defined as $v_{1} \ldots v_{n}=v_{1}^{\prime} \ldots v_{m}^{\prime}$, where $\forall i, v_{i}, v_{i}^{\prime} \in \mathbf{X} \cup \mathcal{C}$. The following theorem identifies the basic forms of word equations. For example, a word equation $f: X_{1}=X_{2} d X_{3} X_{4}$ is equivalent to $\exists X_{k_{1}}, X_{k_{2}} \cdot X_{1}=X_{2} X_{k_{1}} \wedge X_{k_{1}}=$ $d X_{k_{2}} \wedge X_{k_{2}}=X_{3} X_{4}$.

Theorem 2. Word equations and Boolean combinations $(\neg, \wedge$ and $\vee)$ of these equations can be expressed using equations of the form $X_{1}=X_{2} c, X_{1}=c X_{2}, c=X_{1} X_{2}$, $X_{1}=X_{2} X_{3}$, Boolean combinations of such equations and existential quantification. 
Let $f$ be a word equation over $\mathbf{X}=\left\{X_{1}, X_{2}, \ldots, X_{n}\right\}$ and $f[c / X]$ denote a new equation where $X$ is replaced with $c$ for all $X$ that appears in $f$. We say that an $n$ track DFA $M$ under-approximates $f$ if for all $w \in L(M), f\left[\hat{w}[1] / X_{1}, \ldots, \hat{w}[n] / X_{n}\right]$ holds. We say that an $n$-track DFA $M$ over-approximates $f$ if for any $s_{1}, \ldots, s_{n} \in \Sigma^{*}$ where $f\left[s_{1} / X_{1}, \ldots, s_{n} / X_{n}\right]$ holds, there exists $w \in L(M)$ such that for all $1 \leq i \leq$ $n, \hat{w}[i]=s_{i}$. We call $M$ precise with respect to $f$ if $M$ both under-approximates and over-approximates $f$.

Definition 1. A word equation $f$ is regularly expressible if and only if there exists a multi-track DFA $M$ such that $M$ is precise with respect to $f$.

Theorem 3. 1. $X_{1}=X_{2} c, X_{1}=c X_{2}$, and $c=X_{1} X_{2}$ are regularly expressible, as well as their Boolean combinations.

2. $X_{1}=c X_{2}$ is regularly expressible but the corresponding $M$ has exponential number of states in the length of $c$.

3. $X_{1}=X_{2} X_{3}$ is not regularly expressible.

We are able to compute multi-track DFAs that are precise with respect to word equations: $X_{1}=X_{2} c, X_{1}=c X_{2}$, and $c=X_{1} X_{2}$. Since $X_{1}=X_{2} X_{3}$ is not regularly expressible, below, we describe how to compute DFAs that approximate such non-linear word equations. Using the DFA constructions for these four basic forms we can construct multi-track DFAs for all word equations and their Boolean combinations (if the word equation contains a non-linear term then the constructed DFA will approximate the equation, otherwise it will be precise). The Boolean operations conjunction, disjunction and negation can be handled with intersection, union, and complement modulo alignment of the multi-track DFAs, respectively. Existential quantification on the other hand, can be handled using homomorphism, where given a word equation $f$ and a multi-track automaton $M$ such that $M$ is precise with respect to $f$, then the multi-track automaton $M L_{i}$ is precise with respect to $\exists X_{i} . f$ where $M L_{i}$ denotes the result of erasing the $i^{t h}$ track (by homomorphism) of $M$.

Construction of $X_{1}=X_{2} X_{3}$ Since Theorem 3 shows that $X_{1}=X_{2} X_{3}$ is not regularly expressible, it is necessary to construct a conservative (over or under) approximation of $X_{1}=X_{2} X_{3}$. We first propose an over approximation construction for $X_{1}=X_{2} X_{3}$. Let $M_{1}=\left\langle Q_{1}, \Sigma, \delta_{1}, I_{1}, F_{1}\right\rangle, M_{2}=\left\langle Q_{2}, \Sigma, \delta_{2}, I_{2}, F_{2}\right\rangle$, and $M_{3}=$ $\left\langle Q_{3}, \Sigma, \delta_{3}, I_{3}, F_{3}\right\rangle$ accept values of $X_{1}, X_{2}$, and $X_{3}$, respectively. $M=\langle Q,(\Sigma \cup$ $\left.\{\lambda\})^{3}, \delta, I, F\right\rangle$ is constructed as follows.

- $Q \subseteq Q_{1} \times Q_{2} \times Q_{3} \times Q_{3}$,

- $I=\left(I_{1}, I_{2}, I_{3}, I_{3}\right)$,

- $\forall a, b \in \Sigma, \delta\left(\left(r, p, s, s^{\prime}\right),(a, a, b)\right)=\left(\delta_{1}(r, a), \delta_{2}(p, a), \delta_{3}(s, b), s^{\prime}\right)$,

- $\forall a, b \in \Sigma, p \in F_{2}, s \notin F_{3}, \delta\left(\left(r, p, s, s^{\prime}\right),(a, \lambda, b)\right)=\left(\delta_{1}(r, a), p, \delta_{3}(s, b), \delta_{3}\left(s^{\prime}, a\right)\right)$,

- $\forall a \in \Sigma, p \in F_{2}, s \in F_{3}, \delta\left(\left(r, p, s, s^{\prime}\right),(a, \lambda, \lambda)\right)=\left(\delta_{1}(r, a), p, s, \delta_{3}\left(s^{\prime}, a\right)\right)$,

- $\forall a \in \Sigma, p \notin F_{2}, s \in F_{3}, \delta\left(\left(r, p, s, s^{\prime}\right),(a, a, \lambda)\right)=\left(\delta_{1}(r, a), \delta_{2}(p, a), s, s^{\prime}\right)$,

- $F=\left\{\left(r, p, s, s^{\prime}\right) \mid r \in F_{1}, p \in F_{2}, s \in F_{3}, s^{\prime} \in F_{3}\right\}$. 
The intuition is as follows: $M$ traces $M_{1}, M_{2}$ and $M_{3}$ on the first, second and third tracks, respectively, and makes sure that the first and second tracks match each other. After reaching an accepting state in $M_{2}, M$ enforces the second track to be $\lambda$ and starts to trace $M_{3}$ on the first track to ensure the rest (suffix) is accepted by $M_{3} \cdot|Q|$ is $O\left(\left|Q_{1}\right| \times\left|Q_{2}\right| \times\left|Q_{3}\right|+\left|Q_{1}\right| \times\left|Q_{3}\right| \times\left|Q_{3}\right|\right)$. For all $w \in L(M)$, the following hold:

$-\hat{w}[1] \in L\left(M_{1}\right), \hat{w}[2] \in L\left(M_{2}\right), \hat{w}[3] \in L\left(M_{3}\right)$,

$-\hat{w}[1]=\hat{w}[2] w^{\prime}$ and $w^{\prime} \in L\left(M_{3}\right)$,

Note that $w^{\prime}$ may not be equal to $\hat{w}[3]$, i.e., there exists $w \in L(M), \hat{w}[1] \neq$ $\hat{w}[2] \hat{w}[3]$, and hence $M$ is not precise with respect to $X_{1}=X_{2} X_{3}$. On the other hand, for any $w$ such that $\hat{w}[1]=\hat{w}[2] \hat{w}[3]$, we have $w \in L(M)$, hence $M$ is a regular over-approximation of $X_{1}=X_{2} X_{3}$.

Below, we describe how to construct a regular under-approximation of $X_{1}=X_{2} X_{3}$ (which is necessary for conservative approximation of its complement set). We use the idea that if $L\left(M_{2}\right)$ is a finite set language, one can construct the DFA $M$ that satisfies $X_{1}=X_{2} X_{3}$ by explicitly taking the union of the construction of $X_{1}=c X_{3}$ for all $c \in L\left(M_{2}\right)$. If $L\left(M_{2}\right)$ is an infinite set language, we construct a regular underapproximation of $X_{1}=X_{2} X_{3}$ by considering a (finite) subset of $L\left(M_{2}\right)$ where the length is bounded. Formally speaking, for each $k \geq 0$ we can construct $M_{k}$, so that $w \in L\left(M_{k}\right), \hat{w}[1]=\hat{w}[2] \hat{w}[3], \hat{w}[1] \in L\left(M_{1}\right), \hat{w}[3] \in L\left(M_{3}\right), \hat{w}[2] \in L\left(M_{2}\right)$ and $|\hat{w}[2]| \leq k$. It follows that $M_{k}$ is a regular under-approximation of $X_{1}=X_{2} X_{3}$. If $L\left(M_{2}\right)$ is a finite set language, there exists $k$ (the length of the longest accepted word) where $L\left(M_{k}\right)$ is precise with respect to $X_{1}=X_{2} X_{3}$. If $L\left(M_{2}\right)$ is an infinite set language, there does not exist such $k$ so that $L\left(M_{k}\right)$ is precise with respect to $X_{1}=$ $X_{2} X_{3}$, as we have proven non-regularity of $X_{1}=X_{2} X_{3}$.

\section{Symbolic Reachability Analysis}

Our symbolic reachability analysis involves two main steps: forward fixpoint computation and summarization.

Forward Fixpoint Computation The first phase of our analysis is a standard forward fixpoint computation on multi-track DFAs. Each program point is associated with a single multi-track DFA, where each track is associated with a single string variable $X \in \mathbf{X}$. We use $M[l]$ to denote the multi-track automaton at the program label $l$. The forward fixpoint computation algorithm is a standard work-queue algorithm. Initially, for all labels $l, L(M[l])=\emptyset$. We iteratively compute the post-images of the statements and join the results to the corresponding automata. For a stmt in the form: $X:=\operatorname{sexp}$, the post-image is computed as:

$$
\operatorname{Post}(M, \operatorname{stm} t) \equiv\left(\exists X . M \cap \operatorname{Construct}\left(X^{\prime}=\operatorname{sexp},+\right)\right)\left[X / X^{\prime}\right] .
$$

CONSTRUCT $(\exp , b)$ returns the DFA that accepts a regular approximation of exp, where $b \in\{+,-\}$ indicates the direction (over or under, respectively) of approximation if needed. During the construction, we recursively push the negations $(\neg$ ) (and flip the direction) inside to the basic expressions (bexp), and use the corresponding construction 

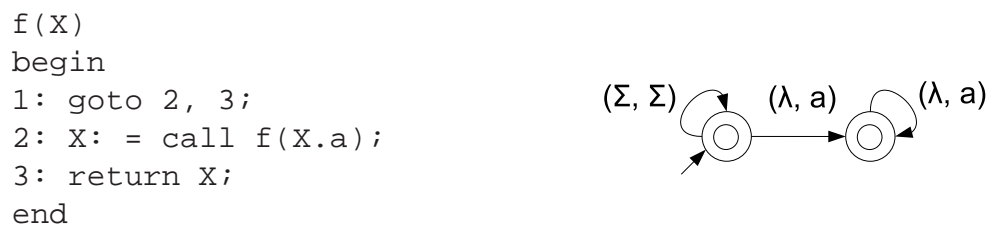

Fig. 2. A function and its summary DFA

of multi-track DFAs discussed in the previous section. We use function summaries to handle function calls. Each function $f$ is summarized as a finite state transducer, denoted as $M_{f}$, which captures the relations among input variables (parameters), denoted as $X_{p}$, and return values. The return values are tracked in the output track, denoted as $X_{o}$. We discuss the generation of the transducer $M_{f}$ below. For a stmt in the form $X:=$ call $f\left(e_{1}, \ldots, e_{n}\right)$, the post-image is computed as:

$$
\operatorname{PosT}(M, s t m t) \equiv\left(\exists X, X_{p_{1}}, \ldots X_{p_{n}} \cdot M \cap M_{I} \cap M_{f}\right)\left[X / X_{o}\right],
$$

where $M_{I}=\operatorname{ConstRUCT}\left(\bigwedge_{i} X_{p_{i}}=e_{i},+\right)$. The process terminates when we reach a fixpoint. To accelerate the fixpoint computation, we extend our automata widening operator [14], denoted as $\nabla$, to multi-track automata. We identify equivalence classes according to specific equivalence conditions and merge states in the same equivalence class $[1,3]$. The following lemma shows that the equality relations among tracks are preserved while widening multi-track automata.

Lemma 1. if $L(M) \subseteq L(x=y)$ and $L\left(M^{\prime}\right) \subseteq L(x=y), L\left(M \nabla M^{\prime}\right) \subseteq L(x=y)$.

Summarization We compute procedure summaries in order to handle procedure calls. We assume parameter-passing with call-by-value semantics and we are able to handle recursion. Each function $f$ is summarized as a multi-track DFA, denoted as $M_{f}$, that captures the relation among its input variables and return values.

Consider the recursive function $f$ shown in Figure 2 with one parameter. $f$ nondeterministically returns its input (goto 3 ) or makes a self call (goto 2) by concatenating its input and a constant $a$. The generated summary for this function is also shown in Figure 2. $M_{f}$ is a 2-track DFA, where the first track is associated with its parameter $X_{p_{1}}$, and the second track is associated with $X_{o}$ representing the return values. The edge $(\Sigma, \Sigma)$ represents a set of identity edges. In other words, $\delta(q,(\Sigma, \Sigma))=q^{\prime}$ means $\forall a \in \Sigma, \delta(q,(a, a))=q^{\prime}$. The summary DFA $M_{f}$ precisely captures the relation $X_{o}=$ $X_{p_{1}} . a^{*}$ between the input variable and the return values.

During the summarization phase, (possibly recursive) functions are summarized as unaligned multi-track DFAs that specify the relations among their inputs and return values. We first build (possibly cyclic) dependency graphs to specify how the inputs flow to the return values. Each node in the dependency graph is associated with an unaligned multi-track DFA that traces the relation among inputs and the value of that node. An unaligned multi-track DFA is a multi-track DFA where $\lambda \mathrm{s}$ might not be right justified. Return values of a function are represented with an auxiliary output track. Given a function $f$ with $n$ parameters, $M_{f}$ is an unaligned $(n+1)$-track DFA, where $n$ 
tracks represent the $n$ input parameters and one track $X_{o}$ is the output track representing the return values. We iteratively compute post images of reachable relations and join the results until we reach a fixpoint. Upon termination, the summary is the union of the unaligned DFAs associated with the return nodes. To compose these summaries at the call site, we also propose an alignment algorithm to align (so that $\lambda$ 's are right justified) an unaligned multi-track DFA.

Once the summary DFA $M_{f}$ has been computed, it is not necessary to reanalyze the body of $f$. To compute the post-image of a call to $f$ we intersect the values of input parameters with $M_{f}$ and use existential quantification to obtain the return values. Let $M$ be a one-track DFA associated with $X$ where $L(M)=\{b\} . \operatorname{Post}(M, X:=$ call $f(X)$ ) returns $M^{\prime}$ where $L\left(M^{\prime}\right)=b a^{*}$ for the example function shown above. As another example, let $M$ be a 2-track DFA associated with $X, Y$ that is precise with respect to $X=Y$. Then $\operatorname{Post}(M, X:=\operatorname{call} f(X))$ returns $M^{\prime}$ which is precise with respect to $X=Y . a^{*}$ precisely capturing the relation between $X$ and $Y$ after the execution of the function call. As discussed above, $M^{\prime}$ is computed by $\left(\exists X, X_{p_{1}} \cdot M \cap\right.$ $\left.M_{I} \cap M_{f}\right)\left[X / X_{o}\right]$, where $L\left(M_{I}\right)=\operatorname{Construct}\left(X_{p_{1}}=X,+\right)$.

\section{Experiments}

We evaluate our approach against three kinds of benchmarks: 1) Basic benchmarks, 2) XSS/SQLI benchmarks, and 3) MFE benchmarks. These benchmarks represent typical string manipulating programs along with string properties that address severe web vulnerabilities.

In the first set, we demonstrate that our approach can prove implicit equality properties of string systems. We wrote two small programs. CheckBranch (B1) has if branch $\left(X_{1}=X_{2}\right)$ and else branch $\left(X_{1} \neq X_{2}\right)$. In the else branch, we assign a constant string $c$ to $X_{1}$ and then assign the same constant string to $X_{2}$. We check at the merge point whether $X_{1}=X_{2}$. In CheckLoop (B2) we assign variables $X_{1}$ and $X_{2}$ the same constant string at the beginning, and iteratively append another constant string to both in an infinite loop. We check whether $X_{1}=X_{2}$ at the loop exit. Let $M$ accept the values of $X_{1}$ and $X_{2}$ upon termination. The equality assertion holds when $L(M) \subseteq L\left(M_{a}\right)$, where $M_{a}$ is Construct $\left(X_{1}=X_{2},-\right)$. We use "-" to construct (under approximation) automata for assertions to ensure the soundness of our analysis. Using multi-track DFAs, we prove the equality property (result "true") whereas we are unable to prove it using single-track DFAs (result "false") as shown in Table 1 (B1 and B2). Though these benchmark examples are simple, to the best of our knowledge, there are no other string analysis tools that can prove equality properties in these benchmarks.

In the second set, we check existence of Cross-Site Scripting (XSS) and SQL Injection (SQLI) vulnerabilities in Web applications with known vulnerabilities. We check whether at a specific program point, a sensitive function may take an attack string as its input. If so, we say that the program is vulnerable with respect to the given attack pattern. To identify XSS/SQLI attacks, we check intersection emptiness against all possible input values that reach a sensitive function at a given program point and the attack strings specified as a regular language. Though one can check such vulnerabilities using single-track DFAs [14], using multi-track automata, we can precisely interpret branch 
conditions, such as $\$ w W w=\$ u r l$, that cannot be precisely expressed using single-track automata, and obtain more accurate characterization of inputs of the sensitive functions. For the vulnerabilities identified in these benchmarks (S1 to S4), we did not observe false alarms that result from the approximation of the branch conditions.

The last set of benchmarks show that the precision that is obtained using multitrack DFAs can help us in removing false alarms generated by single-track automata based string analysis. These benchmarks represent malicious file execution (MFE) attacks. Such vulnerabilities are caused because developers directly use or concatenate potentially hostile input with file or stream functions, or improperly trust input files. We systematically searched web applications for program points that execute file functions, such as include and fopen, whose arguments may be influenced by external inputs. At these program points, we check whether the retrieved files and the external inputs are consistent with what the developers intend. We manually generate a multitrack DFA $M_{v u l}$ that accepts a set of possible violations for each benchmark, and apply our analysis on the sliced program segments. Upon termination, we report that the file function is vulnerable if $L(M) \cap L\left(M_{v u l}\right) \neq \emptyset$. $M$ is the composed DFA of the listed single-track DFAs in the single-track analysis. As shown in Table 1 (M1 to M5), using multi-track DFAs we are able to verify that MFE vulnerabilities do not exist whereas string analysis using single-track DFAs raises false alarms for all these examples.

We have shown that multi-track DFAs can handle problems that cannot be handled by multiple single-track DFAs, but at the same time, they use more time and memory. For these benchmarks, the cost seems affordable. As shown in Table 1, in all tests, the multi-track DFAs that we computed (even for the composed ones) are smaller than the product of the corresponding single-track DFAs. One advantage of our implementation is symbolic DFA representation (provided by the MONA DFA library [5]), in which transition relations of the DFA are represented as Multi-terminal Binary Decision Diagrams (MBDDs). Using the symbolic DFA representation we avoid the potential exponential blow-up that can be caused by the product alphabet. However, in the worst case the size of the MBDD can still be exponential in the number of tracks.

\section{Conclusion}

In this paper, we presented a formal characterization of the string verification problem, investigated the decidability boundary for string systems, and presented a novel verification technique for string systems. Our verification technique is based on forward symbolic reachability analysis with multi-track automata, conservative approximations of word equations and summarization. We demonstrated the effectiveness of our approach on several benchmarks.

\section{References}

1. C. Bartzis and T. Bultan. Widening arithmetic automata. In CAV, pages 321-333, 2004.

2. N. Bjørner, N. Tillmann, and A. Voronkov. Path feasibility analysis for string-manipulating programs. In TACAS, pages 307-321, 2009. 


\begin{tabular}{|c|c|c|c|c|c|c|c|c|}
\hline & \multicolumn{3}{|c}{ Single-track } & \multicolumn{4}{c|}{ Multi-track } \\
\hline Ben & Result & $\begin{array}{c}\text { DFAs/ Composed DFA } \\
\text { state(bdd) }\end{array}$ & $\begin{array}{c}\text { Time } \\
\text { user+sys(sec) }\end{array}$ & $\begin{array}{c}\text { Mem } \\
(\mathrm{kb})\end{array}$ & Result & $\begin{array}{c}\text { DFA } \\
\text { state(bdd) }\end{array}$ & $\begin{array}{c}\text { Time } \\
\text { user+sys(sec) }\end{array}$ & $\begin{array}{c}\text { Mem } \\
(\mathrm{kb})\end{array}$ \\
\hline \hline B1 & false & $15(107), 15(107) / 33(477)$ & $0.027+0.006$ & 410 & true & $14(193)$ & $0.070+0.009$ & 918 \\
\hline B2 & false & $6(40), 6(40) / 9(120)$ & $0.022+0.008$ & 484 & true & $5(60)$ & $0.025+0.006$ & 293 \\
\hline \hline S1 & vul & $2(20), 9(64), 17(148)$ & $0.010+0.002$ & 444 & vul & $65(1629)$ & $0.195+0.150$ & 1231 \\
\hline S2 & vul & $9(65), 42(376)$ & $0.017+0.003$ & 626 & vul & $49(1205)$ & $0.059+0.006$ & 4232 \\
\hline S3 & vul & $11(106), 27(226)$ & $0.032+0.003$ & 838 & vul & $47(2714)$ & $0.153+0.008$ & 2684 \\
\hline S4 & vul & $53(423), 79(633)$ & $0.062+0.005$ & 1696 & vul & $79(1900)$ & $0.226+0.003$ & 2826 \\
\hline \hline M1 & yes & $2(8), 28(208) / 56(801)$ & $0.027+0.003$ & 621 & no & $50(3551)$ & $0.059+0.002$ & 1294 \\
\hline M2 & yes & $2(20), 11(89) / 22(495)$ & $0.013+0.004$ & 555 & no & $21(604)$ & $0.040+0.004$ & 996 \\
\hline M3 & yes & $2(20), 2(20) / 5(113)$ & $0.008+0.002$ & 417 & no & $3(276)$ & $0.018+0.001$ & 465 \\
\hline M4 & yes & $24(181), 2(8), 25(188) / 1201(25949)$ & $0.226+0.025$ & 9495 & no & $181(9893)$ & $0.784+0.07$ & 19322 \\
\hline M5 & yes & $2(8), 14(101), 15(108) / 211(3195)$ & $0.049+0.008$ & 1676 & no & $62(2423)$ & $0.097+0.005$ & 1756 \\
\hline
\end{tabular}

Table 1. Experimental results. DFA(s): the minimized DFA(s) associated with the checked program point. state: number of states. bdd: number of bdd nodes. Benchmark: Application, script (line number). S1: MyEasyMarket-4.1, trans.php (218). S2: PBLguestbook-1.32, pblguestbook.php(1210), S3:Aphpkb-0.71, saa.php(87), and S4: BloggIT 1.0, admin.php (23). M1: PBLguestbook-1.32, pblguestbook.php(536). M2: MyEasyMarket-4.1, prod.php (94). M3: MyEasyMarket-4.1, prod.php (189). M4: phpfusion-6.01, db_backup.php (111). M5: php-fusion-6.01, forums_prune.php (28).

3. A. Bouajjani, P. Habermehl, and T. Vojnar. Abstract regular model checking. In $C A V$, pages 372-386, 2004.

4. A. Bouajjani, B. Jonsson, M. Nilsson, and T. Touili. Regular model checking. In $C A V$, pages 403-418, 2000.

5. BRICS. The MONA project. http://www.brics.dk/mona/.

6. A. S. Christensen, A. Møller, and M. I. Schwartzbach. Precise analysis of string expressions. In $S A S$, pages 1-18, 2003.

7. X. Fu, X. Lu, B. Peltsverger, S. Chen, K. Qian, and L. Tao. A static analysis framework for detecting sql injection vulnerabilities. In COMPSAC, pages 87-96, 2007.

8. C. Gould, Z. Su, and P. Devanbu. Static checking of dynamically generated queries in database applications. In ICSE, pages 645-654, 2004.

9. Y. Minamide. Static approximation of dynamically generated web pages. In $W W W$, pages 432-441, 2005.

10. Open Web Application Security Project (OWASP). Top ten project. http://www . owasp.org/, May 2007.

11. D. Shannon, S. Hajra, A. Lee, D. Zhan, and S. Khurshid. Abstracting symbolic execution with string analysis. In TAICPART-MUTATION, pages 13-22, DC, USA, 2007.

12. G. Wassermann and Z. Su. Static detection of cross-site scripting vulnerabilities. In ICSE, pages 171-180, 2008.

13. F. Yu, M. Alkhalaf, and T. Bultan. Stranger: An automata-based string analysis tool for php. In TACAS, pages 154-157, 2010.

14. F. Yu, T. Bultan, M. Cova, and O. H. Ibarra. Symbolic string verification: An automata-based approach. In SPIN, pages 306-324, 2008.

15. F. Yu, T. Bultan, and O. H. Ibarra. Symbolic string verification: Combining string analysis and size analysis. In TACAS, pages 322-336, 2009.

16. F. Yu, T. Bultan, and O. H. Ibarra. Verification of string manipulating programs using multitrack automata. Technical Report 2009-14, Computer Science Department, University of California, Santa Barbara, August 2009. 